\title{
CRITERIA FOR EXPEDITED ANTIRETROVIRAL INITIATION AND EMERGENCY TRIAGE
}

Few medical interventions for any illness arrest morbidity and mortality as effectively as antiretroviral therapy (ART) does for HIV. Delays result in rapid progression to illness and death. The guidelines below list the patients who qualify for urgent ART, with timeline expectations. In addition, those who do not qualify for 'urgent' ART must receive as rapid an assessment as is possible.

It has become apparent that HIV mortality immediately before initiation of ART accounts for the majority of the deaths that occur in the peri-ART initiation period. The rate of hospitalisation during this period is also high. While the reasons for this situation are complex, at least some of the morbidity and mortality relates to delayed initiation owing to a lack of urgency in staging, referral, counselling and the clinical process of ART initiation.

Anecdotal reports of sites with waiting lists of several months for ART appointments, sites insisting on in-person appointments, delays in staging CD4 counts and difficulty in contacting ART sites, along with unnecessarily protracted counselling, have all been reported to the South African National AIDS Council (SANAC) Treatment Care and Support Technical Task Team. There have even been reports that at several sites (largely hospital sites) the switchboard does not know whether an HIV or ART site exists there, despite the site being fully functional.

Adequate allocated resources are available through the Department of Health for the HIV programme, including the provision of timely ART. No deserving patient should have to experience unreasonable delays for appointments or ART initiation.

The consequence of these delays is delayed initiation of ART with unnecessary mortality and morbidity, and patient frustration at difficult referral procedures, with subsequent loss to follow-up. The delays are often due to a lack of insight into the urgency of initiation of ART. The resulting increased hospitalisation is unnecessary and a massive drain on hospital resources.

\section{LETTER TO HEALTH CARE COLLEAGUES}

\section{Subject: Urgent - expedited ARV initiation guidelines for South African state HIV clinics}

This is the latest version of the expedited antiretroviral initiation guidelines. These have been extensively discussed by the Treatment Task Team of the Programme Implementation Committee of the South African National AIDS Council (SANAC). This Task Team has endorsed the content of the guidelines. The guidelines are now national Department of Health policy.

We recommend that the guidelines are urgently adopted, since we have multiple reports of delays in ART initiation throughout the country, as well as the recent suspension of the ARV initiation programme in the Free State. Recent local research from the universities of Cape Town and and KwaZulu-Natal has emphasised the urgency of initiation of ART in patients with low CD4 counts, those who are very ill and those with tuberculosis.

SANAC is aware of the serious problems recently experienced with the maintenance of ARV supplies in the Free State and the provincial and national Departments of Health are urgently trying to rectify this situation in partnership with SANAC's civil society members. Should you experience problems with drug supplies please refer these to line managers or to the HIV Directorates in the provincial or national DoH. The establishment of a hotline for these reports is currently under discussion and we will inform you once a decision on this has been taken. We encourage private practitioners who experience problems with referrals, or any other health care worker who feels that HIV services are not being adequately provided, to contact the provincial or national $\mathrm{DoH}$ with these reports, which will be urgently investigated. Thank you for your ongoing efforts to provide these critically important services for our HIV-infected patients. Kind regards,

Dr Ashraf Coovadia and Dr François Venter, SANAC Treatment Task Team

Professor Helen Rees, SANACPIC Co-Chairperson 


\section{CD4 STAGING}

- No patient should wait longer than a month for a CD4 count result, from the time blood is taken to the time of clinical assessment and staging and appropriate referral.

- Hospitalised patients and those with tuberculosis (TB) should have this assessment within 1 week of blood being taken.

\section{ART REFERRAL AND INITIATION}

The following patients should not experience a delay in referral to an ART site of more than a week, and must receive their first adherence counselling session immediately upon their first arrival at the clinic. In general, ART should be started within a week of entry to the ART site, unless there are compelling concerns regarding adherence, or an immediate opportunistic infection requires treatment first. In general, any delay should be an exception.

In other words, the interval between referral and ART initiation should never be longer than 2 weeks, unless adherence is considered to be a serious problem.

All patients who fulfil the criteria below, who present on site for the first time, should be seen immediately, even without an appointment. While the patient is in the clinic, take the opportunity to do the first adherence counselling session and to take blood for necessary laboratory tests.

\section{Adults:}

- All adults with a CD4 count $<100$ cells/ $\mu \mathrm{l}$

- All adults with unexplained (after investigation) ongoing loss of weight

- All pregnant women qualifying for ART

- All adults recently hospitalised with an HIV-linked condition (including TB).

\section{Children:}

- All children under 2 years of age

- Any child over 2 years with a CD4 percentage $<15 \%$ or a CD4 count $<100$ cells/ $\mu$ l

All children with unexplained (after investigation) ongoing loss of weight

- All children recently hospitalised with an HIV-linked condition (including TB).

For both adults and children who do NOT fulfi these criteria but qualify for ART, the waiting time should not be longer than a month.

Adherence assessment must be rapid and geared towards addressing any specific issues the individual patient may have.

In certain situations, ART initiation can be done in an inpatient setting. This is especially appropriate where the ART site is hospital based, and hospitalisation is protracted (e.g. a patient started on 2 weeks of amphotericin B for cryptococcal meningitis can be counselled on adherence during hospitalisation, initiated on ART on discharge, and brought back to the clinic directly).

PROCESS IF THE ABOVE CONDITIONS ARE NOT

\section{BEING MET}

The local clinic manager has responsibility for ensuring expedited staging, referral and initiation in sites, including that the switchboard is aware of the clinic's referral processes. All clinic personnel should be trained on which patients require expedited care. If the clinic is not able to function as above, the district manager must investigate and develop an emergency plan to address the situation. Patients should be empowered to know what they can expect from the site, and to make the managers aware if they are experiencing obstruction from clinic personnel. 